\title{
Outcomes in critically ill patients with cancer-related complications
}

\author{
VBL Torres ${ }^{1 *}$, JRL Vassalo ${ }^{1}$, N Spector ${ }^{1}$, FA Bozza ${ }^{2}$, LCP Azevedo ${ }^{3}, J I F ~ S a l l u h^{2}$, M Soares $^{2}$ \\ From ESICM LIVES 2015 \\ Berlin, Germany. 3-7 October 2015
}

\section{Intr}

The number of critically ill patients with malignancies has increased over the past decade and acute complications of cancer or its treatment are one of the most common causes to ICU admission.

\section{Objectives}

Evaluate the clinical characteristics and outcomes of patients admitted to ICUs with complications related to cancer or its treatment in order to identify independent risk factors associated with mortality rates.

\section{Methods}

Secondary analysis of two prospective Brazilians cohort studies. We used logistic regression to identify variables associated with hospital mortality.

\section{Results}

Out of 2028 patients, 456 (23\%) had at least one cancerrelated complication at ICU admission. Compared to those without complications, they had worse performance status (PS) (57\% vs $36 \%$ with PS $\geq 2, P<0.001)$ and had more active disease ( $5 \%$ vs $43 \%, P<0.001)$. The median SOFA score was higher [8 (5-11) vs 6 (4-9), $P<0.001$ ], as well as the need for vasopressors, mechanical ventilation (MV) and dialysis ( $45 \%$ vs $34 \%, 70 \%$ vs $51 \%$ and $12 \%$ vs $8 \%$, respectively) $(P<0.001$ for all). The hospital length of stay (LOS) was similar in both subgroups $(P=0.501)$, but those with complications showed increased ICU mortality ( $47 \%$ vs $27 \%, P<0.001$ ). The most frequent complications were chemotherapy toxicity $(5 \%)$, venous thromboembolism (5\%), respiratory failure by tumor (RFBT) (4\%), gastrointestinal complications by tumor (GCBT) (3\%) and vena cava syndrome (VCS) (2\%). 39/456 patients with

${ }^{1}$ Universidade Federal do Rio de Janeiro, Internal Medicine, Rio de Janeiro, Brazil

Full list of author information is available at the end of the article complications received chemotherapy and/or radiotherapy at ICU, with no mortality difference. Adjusting for the type of admission, hospital LOS prior to ICU and patient's age, the variables independently associated with hospital mortality were: $\mathrm{PS} \geq 2$ [OR $=2.56(2.05-3.20), P<0.001$ ], metastatic solid tumor [OR $=2.11(1.59-2.80), P<0.001]$, high-grade hematologic malignancy [OR $=2.08$ (1.36$3.17), P=0.001]$, higher SOFA score $[\mathrm{OR}=1.16(1.13-$ 1.20), $P<0.001]$, MV [OR $=4.08(3.23-5.15), P<0.001]$, $P<0.001]$, presence of VCS [OR $=3.72(1.10-12.58), P=$ $0.035]$, GCBT [OR $=2.56(1.29-5.09), P=0.007]$ and RFBT $[\mathrm{OR}=1.97(1.04-3.71), P=0.37]$.

\section{Conclusions}

The prognostic impact of cancer-related complications is variable. The presence a severe acute cancer-related complication per se should not guide decisions to admit a patient to the ICU. However, patients presenting with VCS, GCBT and RFBT had worse outcomes.

\section{Authors' details}

${ }^{1}$ Universidade Federal do Rio de Janeiro, Internal Medicine, Rio de Janeiro, Brazil. ' D'Or Institute for Research and Education, Rio de Janeiro, Brazil. ${ }^{3}$ Hospital Sírio Libanês, São Paulo, Brazil.

Published: 1 October 2015

\section{References}

1. Soares M, Darmon M, Salluh Jl, et al: Prognosis of lung cancer patients with life-threatening complications. Chest 2007, 131:840-846.

2. Azoulay $E$, Mokart $D$, Pène $F$, et al: Outcomes of critically ill patients with hematologic malignancies: prospective multicenter data from France and Belgium-a groupe de recherche respiratoire en réanimation oncohématologique study. J Clin Oncol 2013, 31:2810-2818.

3. Puxty $K$, McLoone P, Quasim T, et al: Survival in solid cancer patients following intensive care unit admission. Intensive Care Med 2014, 40:1409-1428.

doi:10.1186/2197-425X-3-S1-A251

Cite this article as: Torres et al: Outcomes in critically ill patients with cancer-related complications. Intensive Care Medicine Experimental 20153 (Suppl 1):A251.

\section{SpringerOpen $^{\circ}$}

(c) 2015 Torres et al.; This is an Open Access article distributed under the terms of the Creative Commons Attribution License (http:// creativecommons.org/licenses/by/4.0), which permits unrestricted use, distribution, and reproduction in any medium, provided the original work is properly cited. 